POS $\quad \begin{aligned} & \text { PROCEEDINGS } \\ & \text { OF SCIENCE }\end{aligned}$

\title{
The QCD coupling $\alpha_{\mathrm{s}}\left(Q^{2}\right)$ at all momentum scales and the elimination of renormalization scale uncertainties
}

\author{
Stanley J. Brodsky* \\ SLAC National Accelerator Laboratory, Stanford University \\ E-mail: sjbtheslac.stanford.edu
}

This contribution discusses two central problems in QCD: (a) the behavior of the QCD running coupling $\alpha_{\mathrm{S}}\left(Q^{2}\right)$ in both the nonperturbative and perturbative domains; and (b) the elimination of perturbative QCD renormalization scale ambiguities.

$\alpha_{s}$ (2019): Workshop on precision measurements of the QCD coupling constant 11-15 February, 2019

Trento, Italy

${ }^{*}$ Speaker. 


\section{Determining the QCD Coupling $\alpha_{\mathrm{s}}\left(Q^{2}\right)$ at all Momentum Scales}

The QCD running coupling $\alpha_{\mathrm{s}}\left(Q^{2}\right)$ sets the strength of the interactions of quarks and gluons as a function of the momentum transfer $Q$. The dependence of the coupling at both small and high momenta is needed to describe hadronic interactions at both long and short distances.

As Grunberg has shown [1], the QCD running coupling can be defined at all momentum scales from a perturbatively calculable physical observable. A particularly useful choice is the effective coupling $\alpha_{g_{1}}^{s}\left(Q^{2}\right)$, which is defined from Bjorken sum rule and is well measured [2]. At high momentum transfer, such "effective charges" satisfy asymptotic freedom, obey the usual pQCD renormalization group equations, and can be related to each other without scale ambiguity by commensurate scale relations [3].

The "dilaton" soft-wall modification of the $\mathrm{AdS}_{5}$ metric $e^{+\kappa^{2} z^{2}}$, together with LF holography, predicts the functional behavior of the running coupling in the small $Q^{2}$ nonperturbative domain [4]: $\alpha_{g_{1}}^{s}\left(Q^{2}\right)=\pi e^{-Q^{2} / 4 \kappa^{2}}$. Measurements of $\alpha_{g_{1}}^{s}\left(Q^{2}\right)$ are remarkably consistent [2] with the Gaussian form predicted by AdSQCD; the best fit gives $\kappa=0.513 \pm 0.007 \mathrm{GeV}$. See Fig. 1 .

Deur, de Téramond, and I $[4,5,6]$ have shown how the parameter $\kappa$, which determines the mass scale of hadrons and Regge slopes in the zero quark mass limit [6], can be connected to the mass scale $\Lambda_{s}$ controlling the evolution of the QCD coupling in the perturbative domain. The high momentum transfer dependence of the coupling $\alpha_{g 1}\left(Q^{2}\right)$ is predicted by pQCD. The matching of the high and low momentum transfer regimes of $\alpha_{g 1}\left(Q^{2}\right)$ - both its value and its slope - then determines a scale $Q_{0}=0.87 \pm 0.08 \mathrm{GeV}$ which sets the interface between perturbative and nonperturbative hadron dynamics. This connection can, in fact, be done for any choice of renormalization scheme, such as the $\overline{\mathrm{MS}}$ scheme.

The result of this perturbative/nonperturbative matching is an effective QCD coupling defined at all momenta. The predicted value of $\Lambda_{\overline{\mathrm{MS}}}=0.339 \pm 0.019 \mathrm{GeV}$ from this analysis agrees well the measured value [7] $\Lambda_{\overline{\mathrm{MS}}}=0.332 \pm 0.017 \mathrm{GeV}$. These results, combined with the AdS/QCD superconformal predictions [8] for hadron spectroscopy, allow one to compute hadron masses in terms of $\Lambda_{\overline{\mathrm{MS}}}: m_{p}=\sqrt{2} \kappa=3.21 \Lambda_{\overline{\mathrm{MS}}}, m_{\rho}=\kappa=2.2 \Lambda_{\overline{\mathrm{MS}}}$, and $m_{p}=\sqrt{2} m_{\rho}$, meeting a challenge proposed by Zee [9]. The mass scale $\kappa$ underlying confinement and hadron masses can thus be connected to the parameter $\Lambda_{\overline{\mathrm{MS}}}$ in the QCD running coupling by matching the nonperturbative prediction to the perturbative QCD regime.

We have also proposed that the value of $Q_{0}$, which marks the interface of nonperturbative and perturbative QCD, can be used to set the factorization scale for DGLAP evolution of hadronic structure functions and ERBL evolution of distribution amplitudes [10]. We have also computed the dependence of $Q_{0}$ on the choice of the effective charge used to define the running coupling and the renormalization scheme used to compute its behavior in the perturbative regime. The use of the scale $Q_{0}$ to resolve the factorization scale uncertainty in structure functions and fragmentation functions, in combination with the scheme-independent principle of maximum conformality (PMC) [11] for setting renormalization scales, can greatly improve the precision of pQCD predictions for collider phenomenology.

The combined approach of light-front holography and superconformal algebra [6] also provides insight into the origin of the QCD mass scale and color confinement. A key observation is the remarkable dAFF principle [12] which shows how a mass scale can appear in the Hamiltonian 

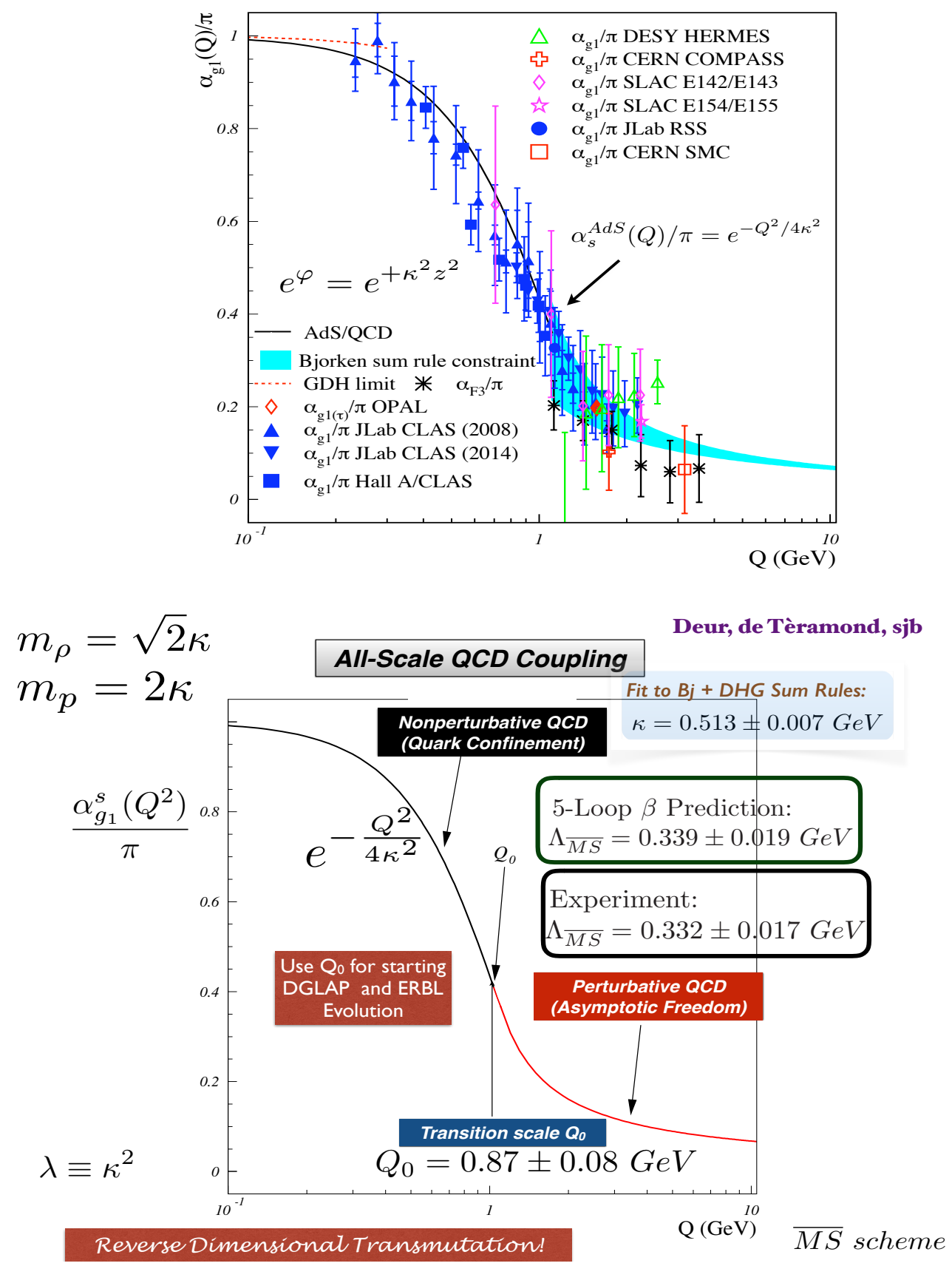

Figure 1: Top: Comparison of the predicted nonperturbative coupling, based on the dilaton $\exp \left(+\kappa^{2} z^{2}\right)$ modification of the $\mathrm{AdS}_{5}$ metric, with measurements of the effective charge $\alpha_{g_{1}}^{s}\left(Q^{2}\right)$, as defined from the Bjorken sum rule. Bottom: Prediction from LF Holography and pQCD for the QCD running coupling $\alpha_{g_{1}}^{s}\left(Q^{2}\right)$ at all scales. The magnitude and derivative of the perturbative and nonperturbative coupling are matched at the scale $Q_{0}$. This matching connects the perturbative scale $\Lambda_{\overline{\mathrm{MS}}}$ in the $\overline{\mathrm{MS}}$ scheme to the nonperturbative mass scale $\kappa=\sqrt{\lambda}$, the mass scale which underlies hadronic masses in QCD. See Ref. [6]. 
and the equations of motion while retaining the conformal symmetry of the action. When one applies the dAFF procedure to chiral QCD, a mass scale $\kappa$ appears which determines universal Regge slopes, hadron masses in the absence of the Higgs coupling, and the mass parameter underlying the Gaussian functional form of the nonperturbative QCD running coupling: $\alpha_{\mathrm{s}}\left(Q^{2}\right) \propto \exp -\left(Q^{2} / 4 \kappa^{2}\right)$. As seen in Fig. 1, this prediction is in remarkable agreement with the effective charge determined from measurements of the Bjorken sum rule.

The potential which underlies color confinement in the effective LF Hamiltonian for the $q \bar{q}$ Fock state of mesons is simply $U\left(\zeta^{2}\right)=\kappa^{4} \zeta^{2}$, a harmonic oscillator potential in the frame-invariant light-front radial variable $\zeta^{2}=b_{\perp}^{2} x(1-x)$. This confinement potential also underlies the spectroscopy and structure of baryons and tetraquarks [6]. The parameter $\kappa$ is not determined in absolute units such as $\mathrm{MeV}$; however, the ratios of mass parameters such as $m_{p} / m_{\rho}=\sqrt{2}$ are predicted. The same potential can also be derived from the anti-deSitter space representation of the conformal group if the $\mathrm{AdS}_{5}$ is action is modified in the fifth dimension $z$ by the dilaton $e^{+\kappa^{2} z^{2}}$. This correspondence is based on light-front holography [13], the duality between dynamics in physical space-time at fixed LF time and five-dimensional AdS space. The predicted light-front wavefunctions can also be used to model "hadronization at the amplitude level" [14].

\section{The Thrust Distribution in Electron-Positron Annihilation using the Principle of Maximum Conformality}

The Principle of Maximum Conformality (PMC) [11, 15, 16, 17, 18] provides a rigorous, systematic way to eliminate renormalization scheme-and-scale ambiguities for perturbative QCD. Since the PMC predictions do not depend on the choice of the renormalization scheme, PMC scalesetting satisfies the principles of renormalization group invariance $[19,20]$.

The PMC provides the underlying principle for extending the Brodsky-Lepage-Mackenzie (BLM) scale-setting method [21] to all orders in pQCD. The essential step is to identify the $\beta$ terms at each order of the $\mathrm{pQCD}$ series. The PMC scales are fixed at every order in $\mathrm{pQCD}$ by absorbing the $\beta$ terms that govern the behavior of the running coupling via the renormalization group equation (RGE). The divergent renormalon terms disappear, and thus the convergence of the pQCD series is greatly improved. The PMC method also sets the renormalization scales for observables that depend on several invariants. The number of active quark flavors $n_{f}$ is set at each order, matching the virtuality of the scattering process. The PMC reduces in the Abelian limit, $N_{C} \rightarrow 0$ [22], to the standard Gell-Mann-Low method [23].

The thrust $(T)$ variable $[24,25]$ is a frequently studied three-jet event shape observables; it is defined as

$$
T=\max _{\vec{n}}\left(\frac{\sum_{i}\left|\vec{p}_{i} \cdot \vec{n}\right|}{\sum_{i}\left|\vec{p}_{i}\right|}\right),
$$

where the sum runs over all particles in the final state, and $\vec{p}_{i}$ denotes the three-momentum of particle $i$. The unit vector $\vec{n}$ is varied to define the thrust direction $\vec{n}_{T}$ by maximizing the sum on the right-hand side. 


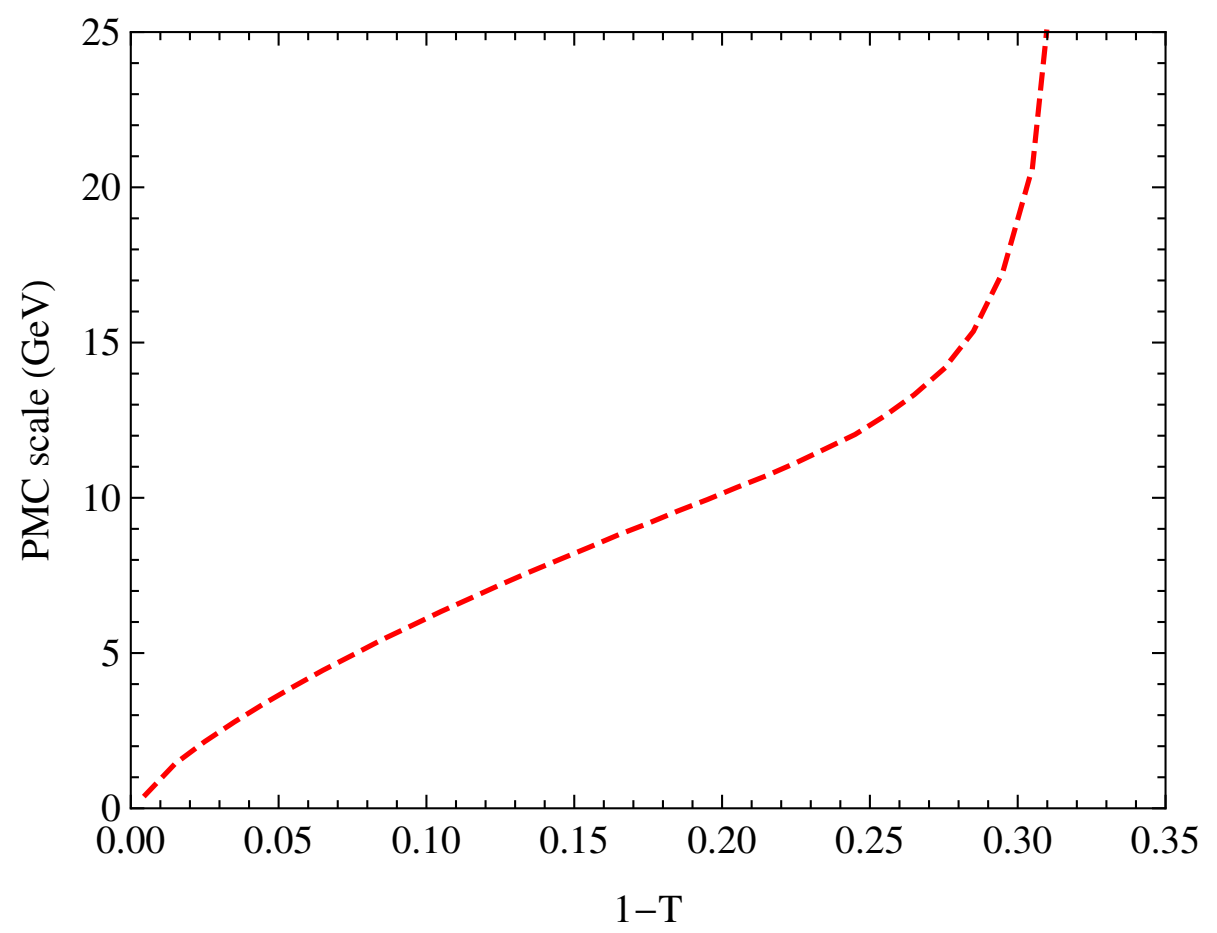

Figure 2: The PMC scales at LL and NLL accuracy for the thrust distribution at $\sqrt{s}=m_{\mathrm{Z}}$.

At the center-of-mass energy $\sqrt{s}$, the differential distribution for thrust variable $\tau=(1-T)$ for renormalization scale $\mu_{r}=\sqrt{s} \equiv Q$ can be written as

$$
\frac{1}{\sigma_{0}} \frac{d \sigma}{d \tau}=A(\tau) a_{s}(Q)+B(\tau) a_{s}^{2}(Q)+\mathscr{O}\left(a_{s}^{3}\right),
$$

where $a_{s}(Q)=\alpha_{\mathrm{s}}(Q) /(2 \pi), \sigma_{0}$ is tree-level hadronic cross section. The $A(\tau), B(\tau), \ldots$ are perturbative coefficients. The experimentally measured thrust distribution is normalized to the total hadronic cross section $\sigma_{h}$,

$$
\frac{1}{\sigma_{h}} \frac{d \sigma}{d \tau}=\bar{A}(\tau) a_{s}(Q)+\bar{B}(\tau) a_{s}^{2}(Q)+\mathscr{O}\left(a_{s}^{3}\right) .
$$

The perturbative coefficients $\bar{A}(\tau)=A(\tau)$, and $\bar{B}(\tau)=B(\tau)-3 / 2 C_{F} A(\tau)$, etc., and their general renormalization scale $\mu_{r}$ dependence $\bar{A}\left(\tau, \mu_{r}\right), \bar{B}\left(\tau, \mu_{r}\right)$ can be restored from the RGE.

In this section, I will review the results of the recent application [26] of PMC scale setting to the thrust distribution by Wang, Wu, de Giustino, and myself. The PMC scale is fixed by absorbing the $\beta_{i}$-terms into the running coupling; it is itself a perturbative expansion series in $\alpha_{\mathrm{s}}$ and in general shows fast $\mathrm{pQCD}$ convergence. A crucial point, as first noted by Gehrmann, Häfliger and Monni [27], is that the pQCD renormalization scale is not a constant; it depends explicitly on the thrust $T$.

In our analysis for the thrust distribution we determine the PMC scale at NLL level by using the pQCD predictions given in Refs. [28, 29]. The inclusion of the NNLO correction only slightly changes the PMC scale determined at NLO level. The PMC scale shows fast $\mathrm{pQCD}$ convergence, as shown explicitly in Fig. 2 . 


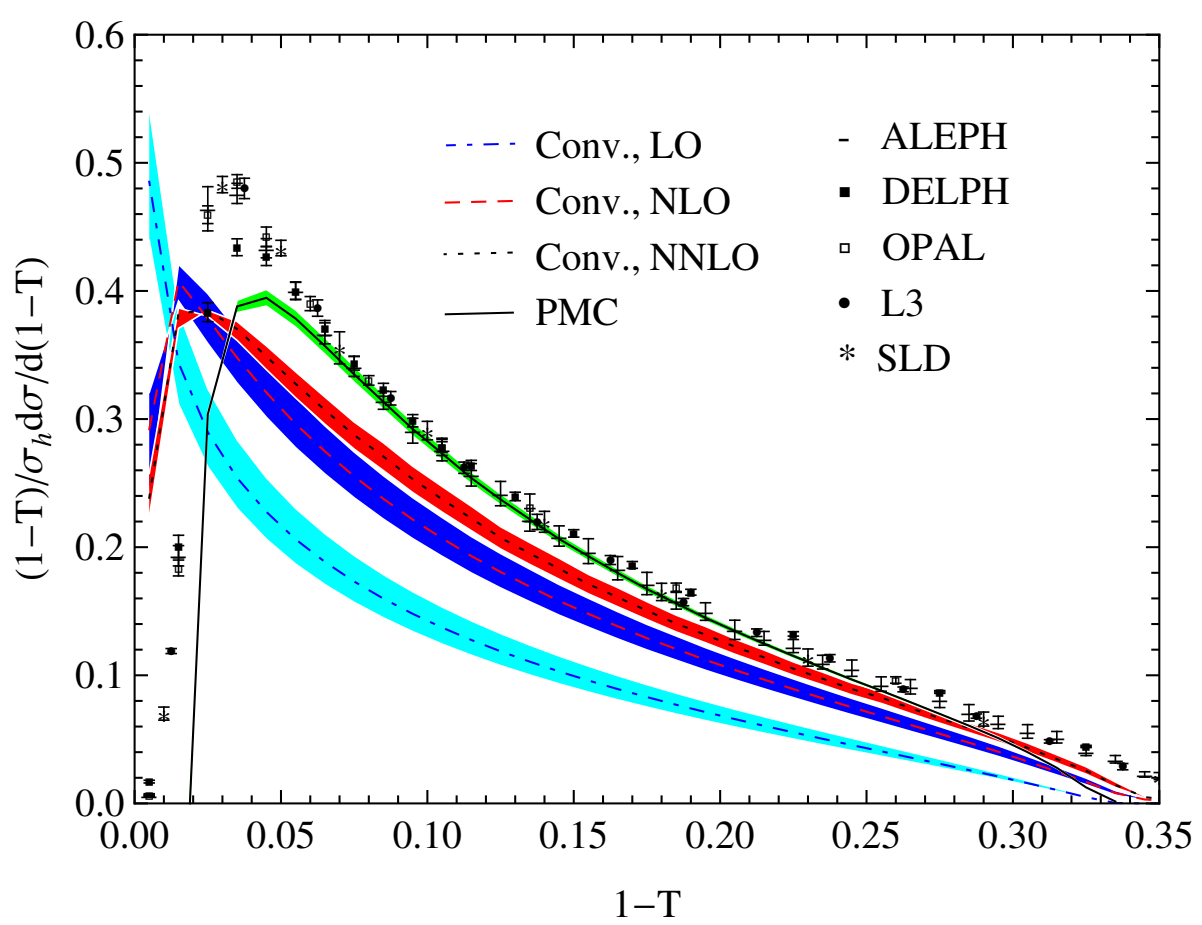

Figure 3: The thrust differential distributions using the conventional (Conv.) and PMC scale settings [26]. The dot-dashed, dashed and dotted lines are the conventional results at LO, NLO and NNLO [28, 29], respectively. The solid line is the PMC result [26]. The bands for the conventional theoretical predictions are obtained by varying $\mu_{r} \in\left[m_{\mathrm{Z}} / 2,2 m_{\mathrm{Z}}\right]$. The PMC prediction eliminates the renormalization scale $\mu_{r}$ uncertainty. Its error band is obtained by using $\alpha_{\mathrm{s}}\left(m_{\mathrm{Z}}\right)=0.1181 \pm 0.0011$ [31]. The experimental data are taken from ALEPH [32], DELPHI [33], OPAL [34], L3 [35], and the SLD [36].

The renormalization scale using conventional scale-setting is simply set at $\mu_{r}=m_{\mathrm{Z}}$. The PMC scale, in contrast, is not a single value, but it monotonically increases with $(1-T)$, reflecting the virtuality of the QCD dynamics. The PMC predictions are in excellent agreement with measurements.

The PMC gives the correct physical behavior of the scale and is bounded in the two-jet region. In addition, the number of active flavors $n_{f}$ changes with $(1-T)$ according to the PMC scale. As the argument of the $\alpha_{\mathrm{s}}$ approaches the two-jet region, the pQCD theory becomes unreliable and non-perturbative effects must be taken into account. One can adopt the predictions from light-front holographic QCD [5] to determine $\alpha_{\mathrm{s}}\left(Q^{2}\right)$ in the low scale domain. The physical behavior of the scale for three-jet processes has also been obtained in Refs. [37, 27]. The soft collinear effective theory determines the thrust distribution at different energy scales and also shows that the two-jet region is affected by non-perturbative effects [30].

A remarkable advantage of using the PMC scale setting is that since the PMC scale varies with $(1-T)$, we can extract directly the strong coupling $\alpha_{\mathrm{s}}$ over a wide range of scales using the experimental data at single center-of-mass-energy, $\sqrt{s}=m_{\mathrm{Z}}$. In this case we have used the most precise data from ALEPH [32]. We have calculated the thrust differential distribution at each bin corresponding to the bins of the experimental data. We can then extract the $\alpha_{\mathrm{s}}$ at different scales bin-by-bin from the comparison of PMC predictions with experimental data. The extracted $\alpha_{\mathrm{s}}$ are 


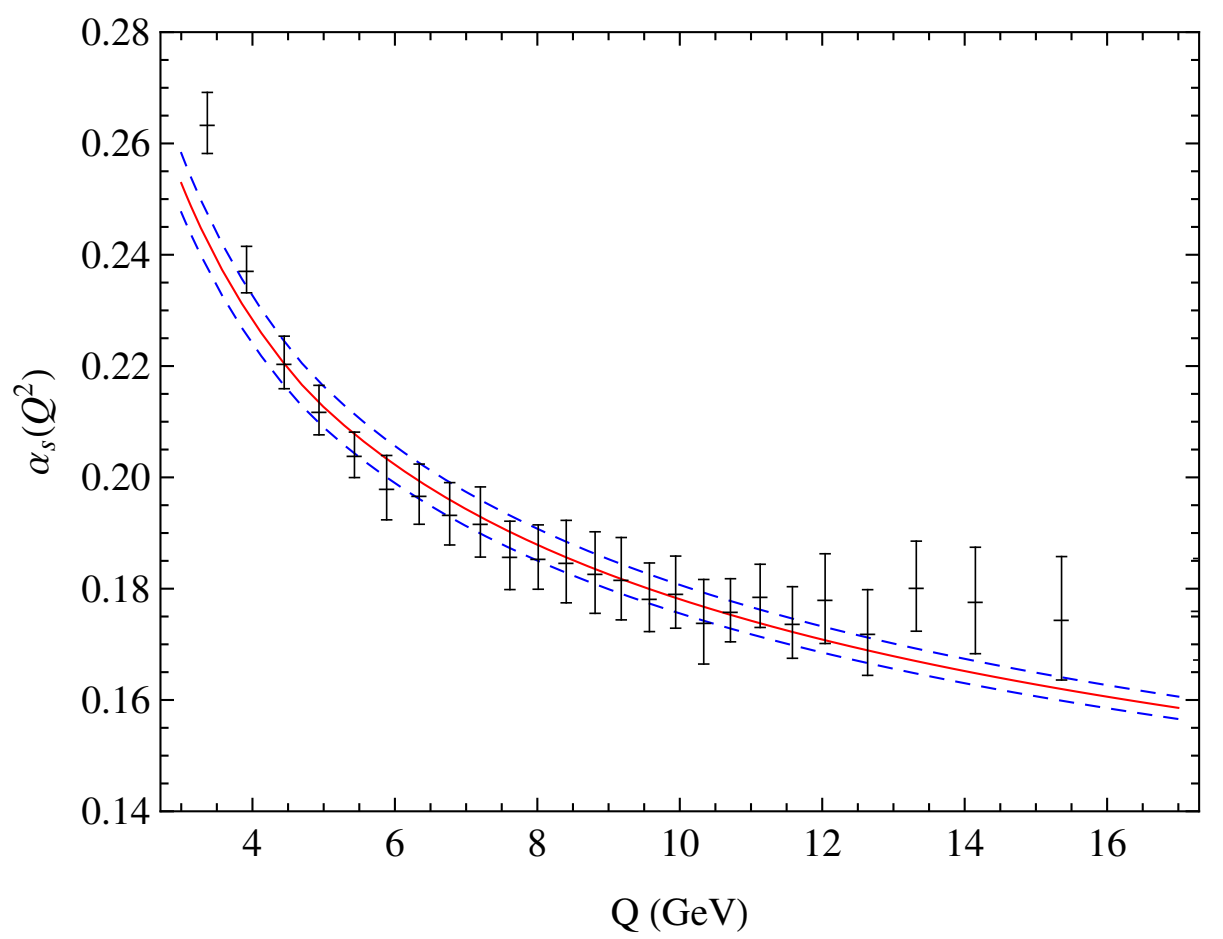

Figure 4: The extracted $\alpha_{\mathrm{s}}\left(Q^{2}\right)$ in the $\overline{\mathrm{MS}}$ scheme from the comparison of PMC predictions with ALEPH data [32] at $\sqrt{s}=m_{\mathrm{Z}}$. The error bars are from the combination of the experimental and theoretical errors. The three lines are the world average evaluated from $\alpha_{\mathrm{S}}\left(m_{\mathrm{Z}}\right)=0.1181 \pm 0.0011$ [31].

explicitly presented in Fig. 4. It shows that in the scale range of $3.5 \mathrm{GeV}<Q<16 \mathrm{GeV}$ (corresponding $(1-T)$ range is $0.05<(1-T)<0.29)$, the extracted $\alpha_{\mathrm{s}}$ are in excellent agreement with the world average evaluated from $\alpha_{\mathrm{s}}\left(m_{\mathrm{Z}}\right)$ [31]. The pQCD calculation corresponds to a parton-level distribution, while the experimental measurements are the hadron-level. Some previous extractions of $\alpha_{\mathrm{s}}$ have applied Monte Carlo generators to correct the effects of hadronization. In our analysis, we have adopted a method similar to [38] in order to extract $\alpha_{\mathrm{s}}$.

In the case of conventional scale setting, the renormalization scale is simply guessed and set at $\mu_{r}=\sqrt{s}=m_{\mathrm{Z}}$, and thus only one value of $\alpha_{\mathrm{S}}$ at scale $m_{\mathrm{Z}}$ can be extracted. The resulting predictions does not fit the measured thrust distribution, and it is incorrect for the QED analog. After using the PMC, we obtain a self-consistent determination of $\alpha_{\mathrm{s}}$ at different scales over a wide range of the thrust distribution. Moreover, since the PMC predictions eliminate the renormalization scale uncertainty, the extracted values for $\alpha_{\mathrm{s}}\left(Q^{2}\right)$ are not plagued by any uncertainty in the choice of $\mu_{r}$. Thus, remarkably, the PMC provides a new way to determine the running of $\alpha_{\mathrm{s}}\left(Q^{2}\right)$ and verify asymptotic freedom from the measurement of jet distributions in $e^{+} e^{-}$annihilation at a single energy of $\sqrt{s}$.

In conclusion, the thrust variable in $e^{+} e^{-}$annihilation provides an ideal platform for testing the QCD. In the case of the conventional scale setting, the predictions are scheme-and-scale dependent, and do not match the experimental results; the extracted coupling constant deviates from the world average. In contrast, after applying PMC scale-setting, we obtain a comprehensive and self-consistent analysis for the thrust measurements, including both the differential distributions 
and the mean values. The PMC scale reflects the virtuality of the QCD dynamics, and it correctly sets the number of active quark flavors $n_{f}$ at every order as a function of the thrust. It allows one to determine $\alpha_{\mathrm{s}}\left(Q^{2}\right)$ at different momentum scales by comparing the PMC predictions with the experiment measured at a single center-of-mass-energy $\sqrt{s}$.

This analysis shows the importance of correct renormalization scale-setting. The PMC method rigorously eliminates an unnecessary theoretical uncertainty for all $\mathrm{pQCD}$ predictions, and it has general applicability for all precision tests of QCD. A recent review of the PMC is given in Ref. [39].

The work of SJB is supported in part by the Department of Energy under contract DE-AC0276SF00515. This contribution (SLAC-PUB-17456) is based on collaborations with Alexandre Deur, Guy de Téramond, Hans Guenter Dosch, Matin Mojaza, Sheng-Quan Wang, Xing-Gang Wu, and Leonard di Giustino.

\section{References}

[1] G. Grunberg, Phys. Lett. B 95, 70 (1980) Erratum: [Phys. Lett. 110, 501 (1982)].

[2] A. Deur, V. Burkert, J. P. Chen and W. Korsch, Phys. Lett. B 650, 244 (2007) [hep-ph/0509113].

[3] S. J. Brodsky and H. J. Lu, Phys. Rev. D 51, 3652 (1995) [hep-ph/9405218].

[4] S. J. Brodsky, G. F. de Téramond and A. Deur, Phys. Rev. D 81, 096010 (2010) [arXiv:1002.3948 [hep-ph]].

[5] A. Deur, S. J. Brodsky and G. F. de Téramond, Phys. Lett. B 750, 528 (2015) [arXiv:1409.5488 [hep-ph]].

[6] S. J. Brodsky, G. F. de Téramond, A. Deur and H. G. Dosch, Few Body Syst. 56, 621 (2015) [arXiv:1410.0425 [hep-ph]].

[7] K. A. Olive et al. [Particle Data Group], Chin. Phys. C 38, 090001 (2014).

[8] H. G. Dosch, G. F. de Téramond and S. J. Brodsky, Phys. Rev. D 91, 085016 (2015) [arXiv:1501.00959 [hep-th]].

[9] A. Zee, Princeton, UK: Princeton Univ. Pr. (2010) 576 pages.

[10] A. Deur, J. M. Shen, X. G. Wu, S. J. Brodsky and G. F. de Téramond, Phys. Lett. B 773, 98 (2017) [arXiv:1705.02384 [hep-ph]].

[11] M. Mojaza, S. J. Brodsky and X. G. Wu, Phys. Rev. Lett. 110, 192001 (2013).

[12] V. de Alfaro, S. Fubini and G. Furlan, Nuovo Cim. A 34, 569 (1976).

[13] G. F. de Téramond and S. J. Brodsky, Phys. Rev. Lett. 102, 081601 (2009) [arXiv:0809.4899 [hep-ph]].

[14] S. J. Brodsky and G. F. de Téramond, arXiv:0901.0770 [hep-ph].

[15] S. J. Brodsky and X. G. Wu, Phys. Rev. D 85, 034038 (2012).

[16] S. J. Brodsky and X. G. Wu, Phys. Rev. Lett. 109, 042002 (2012).

[17] S. J. Brodsky and L. Di Giustino, Phys. Rev. D 86, 085026 (2012).

[18] S. J. Brodsky, M. Mojaza and X. G. Wu, Phys. Rev. D 89, 014027 (2014). 
[19] S. J. Brodsky and X. G. Wu, Phys. Rev. D 86, 054018 (2012).

[20] X. G. Wu, Y. Ma, S. Q. Wang, H. B. Fu, H. H. Ma, S. J. Brodsky and M. Mojaza, Rept. Prog. Phys. 78, 126201 (2015).

[21] S. J. Brodsky, G. P. Lepage and P. B. Mackenzie, Phys. Rev. D 28, 228 (1983).

[22] S. J. Brodsky and P. Huet, Phys. Lett. B 417, 145 (1998).

[23] M. Gell-Mann and F. E. Low, Phys. Rev. 95, 1300 (1954).

[24] S. Brandt, C. Peyrou, R. Sosnowski and A. Wroblewski, Phys. Lett. 12, 57 (1964).

[25] E. Farhi, Phys. Rev. Lett. 39, 1587 (1977).

[26] S. Q. Wang, S. J. Brodsky, X. G. Wu and L. Di Giustino, arXiv:1902.01984 [hep-ph].

[27] T. Gehrmann, N. Häfliger and P. F. Monni, Eur. Phys. J. C 74, 2896 (2014).

[28] A. Gehrmann-De Ridder, T. Gehrmann, E. W. N. Glover and G. Heinrich, Phys. Rev. Lett. 99, 132002 (2007); JHEP 0712, 094 (2007); Comput. Phys. Commun. 185, 3331 (2014).

[29] S. Weinzierl, Phys. Rev. Lett. 101, 162001 (2008); JHEP 0906, 041 (2009).

[30] R. Abbate, M. Fickinger, A. H. Hoang, V. Mateu and I. W. Stewart, Phys. Rev. D 83, 074021 (2011).

[31] M. Tanabashi et al. [Particle Data Group], Phys. Rev. D 98, 030001 (2018).

[32] A. Heister et al. [ALEPH Collaboration], Eur. Phys. J. C 35, 457 (2004).

[33] J. Abdallah et al. [DELPHI Collaboration], Eur. Phys. J. C 29, 285 (2003).

[34] G. Abbiendi et al. [OPAL Collaboration], Eur. Phys. J. C 40, 287 (2005).

[35] P. Achard et al. [L3 Collaboration], Phys. Rept. 399, 71 (2004).

[36] K. Abe et al. [SLD Collaboration], Phys. Rev. D 51, 962 (1995).

[37] G. Kramer and B. Lampe, Z. Phys. A 339, 189 (1991).

[38] T. Becher and M. D. Schwartz, JHEP 0807, 034 (2008).

[39] X. G. Wu, J. M. Shen, B. L. Du, X. D. Huang, S. Q. Wang and S. J. Brodsky, arXiv:1903.12177 [hep-ph]. 